\title{
Avoidance of Radiation Damage in Vibrational-Mode Energy-Loss Spectroscopy.
}

\author{
R.F. Egerton ${ }^{1}$, P. Rez ${ }^{2}$ and P.A. Crozier ${ }^{3,4}$ \\ 1. Physics Department, University of Alberta, Edmonton, Canada T6G 2E1. \\ 2. Department of Physics, Arizona State University, Tempe, AZ 85287, USA. \\ 3. Center for Solid State Science, Arizona State University, Tempe, AZ 85287, USA. \\ ${ }^{4}$ School for Engineering of Matter, Transport and Energy, ASU, Tempe, AZ 85287, USA.
}

New-generation monochromator systems incorporated into transmission electron microscopes allow electron energy-loss spectroscopy (EELS) to be performed on small regions of a thin specimen with an energy resolution in the range $10-50 \mathrm{meV}$. This enhanced performance will be highly useful in characterizing electronic properties of materials (e.g. bandgap studies in semiconductors) and it also makes possible the study of vibrational (phonon) modes of energy loss (vibEELS), which can provide information about the nature of the chemical bonds present in the specimen.

The vibrational-mode peaks occur mostly in the energy range $0.1-0.2 \mathrm{eV}$. Although some high-angle component is expected [2 -4$]$, the recorded signal arises largely from dipole interactions that involve scattering angles below $1 \mathrm{mrad}$ [5], implying that the interaction is delocalized over distances of many $\mathrm{nm}$. Although this delocalization reduces the spatial resolution of vibEELS analysis, it offers the possibility of aloof-mode measurement [6] with the electron probe tens of $\mathrm{nm}$ beyond the edge of the specimen, as demonstrated experimentally [1]. In this mode, electronic transitions within the specimen are only weakly excited, so radiation damage to the specimen might be expected to be minimal. Avoidance of radiation damage is important because many of the interesting vibEELS specimens are beam-sensitive, which further limits the spatial resolution of the analysis [5].

We have simulated the signal and damage in aloof mode by taking the energy-loss probability as:

$$
\mathrm{d} P / \mathrm{d} E=t e^{2}\left(2 \pi^{2} \varepsilon_{0} h^{2} v^{2}\right)^{-1} \operatorname{Im}\{-2 /[\varepsilon(E)+1]\} \mathrm{K}_{0}[4 \pi r E /(\gamma v h)]
$$

where $t$ is the specimen thickness, $v$ is the incident-electron speed, $\varepsilon(E)$ is the relative permittivity of the specimen at an energy loss $E$, and $r$ is distance of the probe from the edge of the specimen. The modified Bessel function $\mathrm{K}_{0}$ represents delocalization of the signal, extending over many nm as shown in Fig. 1.

Equation (1) applies equally to the electronic excitations in the specimen that give rise to radiolytic damage but because of the higher values of $E$, the delocalization is much less; see Fig. 1. In fact, we might expect that radiolysis requires an energy exchange of several $\mathrm{eV}$, as evidenced by the fact that polymers can be degraded by ultraviolet radiation but not by visible or infrared light. If this energy threshold is $5 \mathrm{eV}$, for example, Fig. 1 suggests that a probe placed at least $20 \mathrm{~nm}$ from the edge of the sample will excite few of the electronic excitations that lead to damage but still generate a substantial vibrational-loss signal. Evaluation of the energy-loss function in Eq.(1) allows us to simulate the effect of changing the threshold energy, for comparison with measurements of $\mathrm{d} P / \mathrm{d} E$ as a function of $r$.

The response $\mathrm{d} P / \mathrm{d} E$ can be thought of as the integral of a point-spread function $\mathrm{d} P / \mathrm{d} A$ over nearby areas $A$ of the specimen. If $\mathrm{d} P / \mathrm{d} A \propto 1 / r^{2}$ over most of its range [7], $\mathrm{d} P / \mathrm{d} E \propto \int(\mathrm{d} P / \mathrm{d} A) \mathrm{d} A \propto \int\left(1 / r^{2}\right) r \mathrm{~d} r=$ 
$\log (r)$ and this behavior is confirmed by plotting $\mathrm{K}_{0}$ against $\log (r)$, as in Fig. $1 \mathrm{~b}$. The lower limit $r_{\min }$ of this approximation is determined by the probe diameter and cutoff of the Lorentzian angular distribution of scattering. The upper limit $r_{\max }$ is set by dynamical screening (Bohr adiabatic limit) and gives rise to the curvature seen in Fig. 1b, which suggests $r_{\max } \sim 1000 \mathrm{~nm}$ for a $0.15 \mathrm{eV}$ peak and $60 \mathrm{keV}$ electrons. So we might expect some vibrational-loss signal to arise from material lying as far as $1 \mu \mathrm{m}$ from the edge of the specimen, with half the signal generated within about $30 \mathrm{~nm}$ of the edge, assuming $\log (\mathrm{r})$ behavior.

In conclusion, it appears that radiolysis damage can be almost completely avoided by positioning an electron probe at least $20 \mathrm{~nm}$ from the edge of a specimen, assuming that the probe has no aberration tails, that phonon excitation is non-damaging and that radiolysis requires a minimum energy transfer of a few $\mathrm{eV}$. The spatial resolution of the vibrational-loss signal will then be some tens of $\mathrm{nm}$, somewhat better than the dose-limited resolution (DLR) for the most radiation-sensitive organic materials $[5,8]$.

\section{References:}

[1] OL Krivanek et al, Nature 514 (2014), p. 209.

[2] P Rez, Microsc . Microanal. 20 (2014), p. 671.

[3] C Dwyer, Phys. Rev. B 89 (2014), p. 054103.

[4] P Cueva and DA Muller, Microsc . Microanal. 20, suppl. 3 (2014), p. 590.

[5] RF Egerton, Microsc. Microanal. 20 (2014), p. 658.

[6] A Howie, Ultramicroscopy 11 (1983), p. 141.

[7] RF Egerton, "EELS in the Electron Microscope", $3^{\text {rd }}$ edition, (Springer, New York) p.226.

[8] RFE acknowledges funding from the Natural Sciences and Engineering Research Council of Canada and wishes to thank Michael Bergen for his advice on Matlab programming.

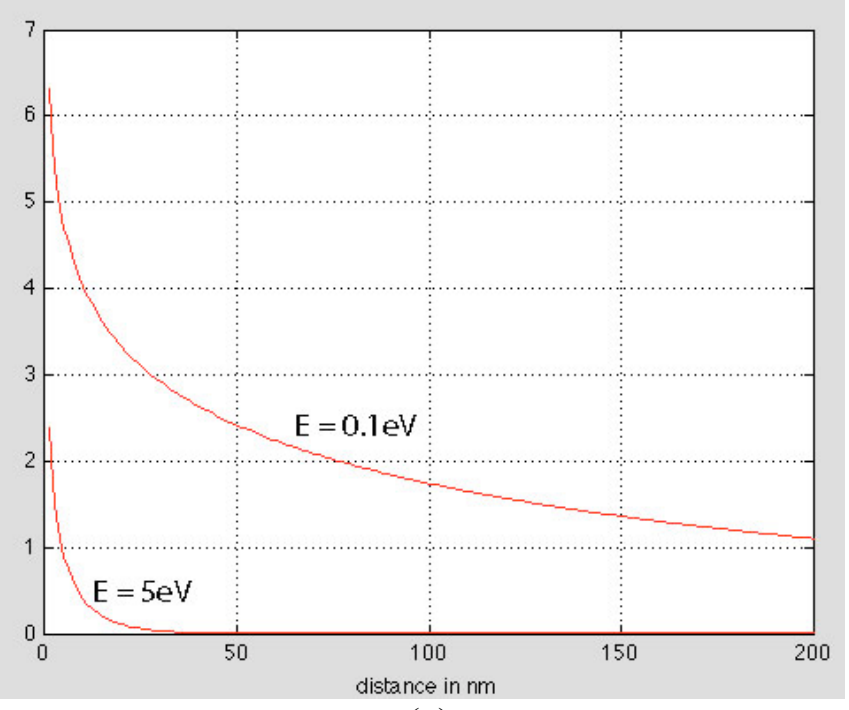

(a)

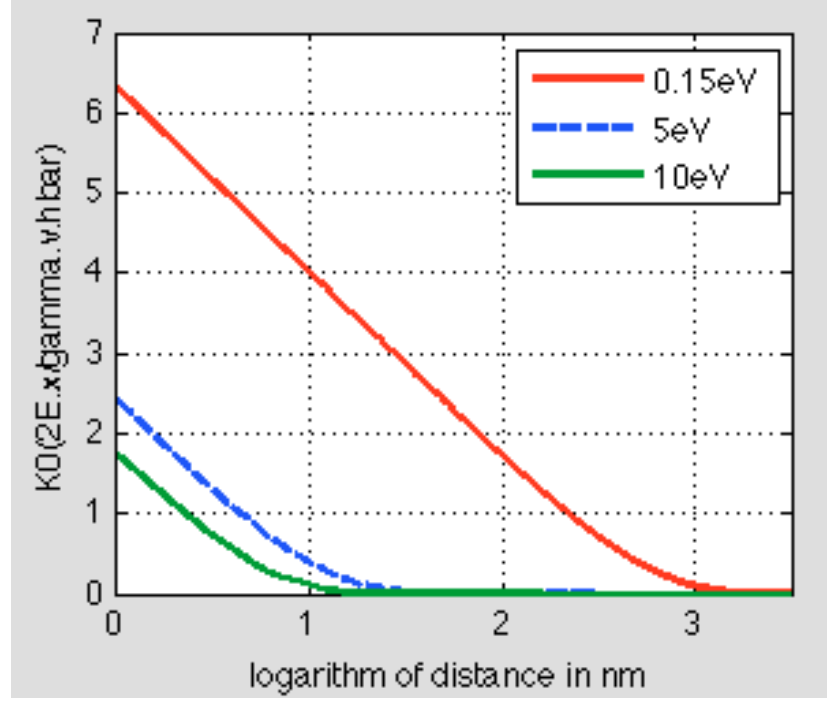

(b)

Figure 1. (a) $\mathrm{K}_{0}$ function of Eq.(1), showing spectral intensity as a function of distance $r$ of the probe from the edge of the specimen, for a vibrational peak at $0.1 \mathrm{eV}$ and an electronic excitation at $5 \mathrm{eV}$ loss, assuming a STEM operating at $60 \mathrm{kV}$. (b) $\mathrm{K}_{0}$ function plotted against $\log _{10}(r)$, for a $0.15 \mathrm{eV}$ vibrational peak and for electronic energy losses of $5 \mathrm{eV}$ and $10 \mathrm{eV}$, assuming an incident energy of $60 \mathrm{keV}$. 